Prepared for the U.S. Department of Energy under Contract DE-AC05-76RL01830

\title{
GUM Analysis for TIMS Isotopic Ratios in BEPO Graphite Qualification Samples, Round 2
}

DC Gerlach BD Reid

PG Heasler

January 2009

Pacific Northwest

NATIONAL LABORATORY 


\title{
DISCLAIMER
}

This report was prepared as an account of work sponsored by an agency of the United States Government. Neither the United States Government nor any agency thereof, nor Battelle Memorial Institute, nor any of their employees, makes any warranty, express or implied, or assumes any legal liability or responsibility for the accuracy, completeness, or usefulness of any information, apparatus, product, or process disclosed, or represents that its use would not infringe privately owned rights. Reference herein to any specific commercial product, process, or service by trade name, trademark, manufacturer, or otherwise does not necessarily constitute or imply its endorsement, recommendation, or favoring by the United States Government or any agency thereof, or Battelle Memorial Institute. The views and opinions of authors expressed herein do not necessarily state or reflect those of the United States Government or any agency thereof.

\author{
PACIFIC NORTHWEST NATIONAL LABORATORY \\ operated by \\ BATTELLE \\ for the \\ UNITED STATES DEPARTMENT OF ENERGY \\ under Contract DE-ACO5-76RL01830
}

Printed in the United States of America

Available to DOE and DOE contractors from the

Office of Scientific and Technical Information,

P.O. Box 62, Oak Ridge, TN 37831-0062;

ph: (865) 576-8401

fax: (865) 5765728

email: reports@adonis.osti.gov

\footnotetext{
Available to the public from the National Technical Information Service,

U.S. Department of Commerce, 5285 Port Royal Rd., Springfield, VA 22161

ph: (800) 553-6847$$
\text { fax: (703) 605-6900 }
$$

email: orders@nits.fedworld.gov

online ordering: http://www.ntis.gov/ordering.htm
} 


\section{GUM Analysis for TIMS Isotopic Ratios in BEP0 Graphite Qualification Samples, Round 2}

DC Gerlach

PG Heasler

BD Reid

January 2009

Prepared for

the U.S. Department of Energy

under Contract DE-AC05-76RL01830

Pacific Northwest National Laboratory

Richland, Washington 99352 



\subsection{Sample Receipt and Preparation}

In May 2007, one set of three samples from NBL were addressed to Steve Petersen for TIMS analysis, and included BEP0 samples numbered 27008, 30986, and 50846. All cores were trimmed by tooling, and lightly cleaned by $\mathrm{CO}_{2}$ pellet blasting. Small discs were cut from the second set of samples for SIMS analysis, with the remainder of each used for TIMS preparation.

Three GIRM Phase III QA samples were received from NBL in August 2008, and consisted of UCAR graphite plugs doped with solutions containing uranium and plutonium. The three samples received at PNNL for TIMS analysis were numbered P3-51C, P3-52A, and P3-60C. These were not trimmed or prepared in the manner above, but the as-received samples were immediately ashed, as in step 1 below.

The general sample preparation procedure for TIMS analysis includes the following steps:

1. Samples ashed and ash acid digested, ending up in $\mathrm{HCl}$. A $20 \%$ aliquot is kept in reserve for processing or replicates if needed.

2. $40 \%$ of ash solution taken for unspiked $U$ separation and TIMS analysis; ${ }^{233} \mathrm{U}{ }^{238} \mathrm{U}$ ratios determined by TIMS analysis rather than ICPMS analyses as used earlier.

3. $40 \%$ spiked with mixed ${ }^{233} \mathrm{U}+{ }^{244} \mathrm{Pu}$ spike; $\mathrm{Pu}-244$ spike amounts were chosen to be appropriate for the sample based on either the SIMS results for B isotopes or the unspiked U isotope ratios, to minimize spike correction on minor $\mathrm{Pu}$ isotopes, and because $\mathrm{Pu}$ contents were expected to vary by up to 300 -fold. This is based on our accumulated experience with the BEP0 samples, and may be useful in other, future sample studies

4. Portion of separated spiked $U$ fraction aliquotted for Pu TIMS analysis and additional U TIMS analysis based on observed $U$ total contents, $2 \mathrm{ng} \mathrm{U}$ usual amount preferred, 1 to $3 \mathrm{pg} \mathrm{Pu}$ preferred (more for low burnup samples).

5. Total U contents calculated based on sample and aliquot weights, and spiked and unspiked ${ }^{233} \mathrm{U} /{ }^{238} \mathrm{U}$ ratios, since many samples contain ${ }^{233} \mathrm{U}$ already. Total Pu contents determined based on measured amount of ${ }^{244} \mathrm{Pu}$ added.

The three NBL BEP0 samples were prepared following these steps, resulting in both spiked and unspiked $\mathrm{U}$ fractions analyzed by TIMS. Unfortunately, $\mathrm{U}$ from both the spiked and unspiked separations was lost, and determination of $U$ contents was not possible. The reserved $20 \%$ fraction for each sample was later processed to obtain more of the $\mathrm{U}$ for TIMS analyses.

The three ('non-BEP0') fabricated QA samples were not split following our usual procedure above, and instead $50 \%$ of the sample solution was processed and combined with the $\mathrm{U}$ and $\mathrm{Pu}$ spikes, and $50 \%$ used for unspiked separations and analyses. Separations are completed and preliminary results for these samples will be available soon, but are not included in this report. 


\subsection{TIMS Analysis}

Separated uranium fractions for TIMS analysis are prepared by solution loading using a nanopipettor mounted in a micromanipulator onto carburized Re filaments. Separated plutonium fractions are equilibrated with single anion resin beads that are loaded onto carburized Re filaments to make a better point source for thermal ion emission. Data are acquired on the mass spectrometer in peak-switching cycles, with each cycle including a scan up and a scan down the designated range of masses. Counting times for each mass are chosen to improve counting statistics, and are different for $\mathrm{U}$ and $\mathrm{Pu}$ acquisitions.

For spiked uranium fractions, the chosen counting times are:

\begin{tabular}{|l|c|c|c|c|c|c|c|}
\hline Mass & 233 & 234 & 235 & 236 & 238 & 240 & 243 \\
\hline Time(sec) & 3 & 12 & 6 & 12 & 3 & 3 & 6 \\
\hline
\end{tabular}

For unspiked uranium fractions, the chosen counting times are:

\begin{tabular}{|l|c|c|c|c|c|c|c|}
\hline Mass & 233 & 234 & 235 & 236 & 238 & 240 & 243 \\
\hline Time(sec) & 12 & 9 & 6 & 12 & 3 & 3 & 6 \\
\hline \hline
\end{tabular}

For plutonium fractions, the chosen counting times are:

\begin{tabular}{|l|c|c|c|c|c|c||}
\hline Mass & 239 & 240 & 241 & 242 & 243 & 244 \\
\hline Time(sec) & 3 & 6 & 12 & 12 & 5 & 7 \\
\hline
\end{tabular}

Run data and results generated by the TIMS mass spectrometer data acquisition routine produce a good initial estimate of run quality and analysis precision. In these preliminary run results, uranium isotope ratio measurements are corrected for measurement mass bias based on results obtained for a natural $\mathrm{U}$ standard NBS 950a. Plutonium isotope ratio results are not corrected for mass bias.

\subsection{Preliminary TIMS Data Reduction}

Resulting or 'raw' mass spec data files are reduced using an in-house routine, which subtracts spike/tracer contributions on other isotopes, and which also generates cycle-by-cycle raw count tables and interpolated count rates. The latter were used in some portions of the GUM analysis. The $2 \sigma$ uncertainties reported for all isotope ratios below are preliminary in a sense, having been generated by our in-house offline data reduction program, whereas the GUM uncertainty analysis includes a more complete estimate of measurement uncertainty. The tables also include the internal sample log numbers assigned to each sample for record-keeping. The values reported below are not yet corrected for measurement mass bias. 


\begin{tabular}{|c|c|c|c|c|c|c|c|}
\hline sample & PNNL No. & ${ }^{234} \mathrm{U} /{ }^{238} \mathrm{U}$ & 2 sigma & ${ }^{235} \mathrm{U} /{ }^{236} \mathrm{U}$ & 2 sigma & ${ }^{236} \mathrm{U} /{ }^{238} \mathrm{U}$ & 2 sigma \\
\hline 27008 & 85238 & 0.000224 & 0.000002 & 0.005062 & 0.000026 & 0.000365 & 0.000004 \\
\hline 30986 & 85239 & 0.000246 & 0.000003 & 0.004803 & 0.000017 & 0.000400 & 0.000004 \\
\hline 50846 & 85240 & 0.000108 & 0.000003 & 0.005799 & 0.000020 & 0.000231 & 0.000003 \\
\hline
\end{tabular}

\begin{tabular}{|c|c|c|c|c|c|c|c|}
\hline sample & PNNL No. & ${ }^{240} \mathrm{Pu} /{ }^{239} \mathrm{Pu}$ & 2 sigma & ${ }^{241} \mathrm{Pu} /{ }^{239} \mathrm{Pu}$ & 2 sigma & ${ }^{242} \mathrm{Pu} /{ }^{239} \mathrm{Pu}$ & 2 sigma \\
\hline 27008 & 85393 & 0.12505 & 0.00030 & 0.002127 & 0.000020 & 0.001039 & 0.000016 \\
\hline 30986 & 85395 & 0.13741 & 0.00031 & 0.002471 & 0.000021 & 0.001364 & 0.000017 \\
\hline 50846 & 85394 & 0.07334 & 0.00018 & 0.000764 & 0.000012 & 0.000210 & 0.000006 \\
\hline
\end{tabular}

\subsection{GUM Analyses of TIMS Results}

All notations and calculations used in the GUM analyses and in tables below are the same as used in the previous GUM report for BEP0 TIMS results.

Uncertainties for the concentrations and isotope ratios of the $\mathrm{U}$ or Pu tracers or 'spikes' were not directly used in GUM uncertainty analyses. No new independent measurements or recalibrations of tracers were performed during the present study. Results of recent or past such measurements can be provided separately upon request, if there is interest. Instead, the amounts of ion counts estimated contributed by the tracer for all isotopes are used in the GUM uncertainty tables for $\mathrm{U}$ and Pu results.

The TIMS mass spectrometer which has been continuously used in this project was down for a period of several months finally ending in July 2008. At that time, a number of tests and standard reference materials were run for operational and quality checks. As a result of this effort, new data for Pu reference standard CRM 138 was acquired and was used to estimate mass bias correction factors (CFs) for these sample results. Recent analyses of natural U standard NBS 950a were also used to estimate mass bias correction factors, however, 'CFs' for ${ }^{233} \mathrm{U} /{ }^{238} \mathrm{U}$ and ${ }^{236} \mathrm{U} /{ }^{238} \mathrm{U}$ ratios are not available yet, since this particular standard lacks any ${ }^{233} \mathrm{U}$ or ${ }^{236} \mathrm{U}$. In the future, we plan to acquire depleted $\mathrm{U}$ reference standards for routine use and for measurement of mass bias factors for all of the $U$ isotope ratios. The uncertainty contributions for CFs were estimated in the GUM results below using these newer and more recent results on measurement reference standards. 


\begin{tabular}{|l|l|l|l|l|l|l|}
\hline \multicolumn{2}{|l|}{ Pu GUM Tables For Sample 85393 (27008) } & & & & \\
\hline${ }^{240} \mathrm{Pu} /{ }^{239} \mathrm{Pu}$ & & & & & & \\
\hline Quantity & Est & $\% \mathrm{RE}$ & dof & Sensit & Uncert & Index \\
\hline R.240.239 & 0.125 & 0.156 & 37.6 & $1.00 \mathrm{E}+00$ & $1.95 \mathrm{E}-04$ & $8.80 \mathrm{E}+01$ \\
\hline CF.240.239 & 1 & 0.0576 & 31 & $1.25 \mathrm{E}-01$ & $7.20 \mathrm{E}-05$ & $1.20 \mathrm{E}+01$ \\
\hline b.tot.239 & 0.658 & 27.9 & Inf & $8.80 \mathrm{E}-07$ & $1.61 \mathrm{E}-07$ & $6.03 \mathrm{E}-05$ \\
\hline b.tot.240 & 3.66 & 5.05 & Inf & $-2.06 \mathrm{E}-05$ & $-3.81 \mathrm{E}-06$ & $3.37 \mathrm{E}-02$ \\
\hline Cor & NA & NA & NA & NA & $-1.11 \mathrm{E}-06$ & $-2.83 \mathrm{E}-03$ \\
\hline Result & 0.125 & 0.166 & 47.5 & NA & NA & NA \\
\hline${ }^{241} \mathrm{Pu} /{ }^{239} \mathrm{Pu}$ & & & & & & \\
\hline R.241.239 & 0.00211 & 0.527 & 37.2 & $1.03 \mathrm{E}+00$ & $1.15 \mathrm{E}-05$ & $3.39 \mathrm{E}+01$ \\
\hline CF.241.239 & 1.03 & 0.715 & 31 & $2.11 \mathrm{E}-03$ & $1.56 \mathrm{E}-05$ & $6.24 \mathrm{E}+01$ \\
\hline b.tot.239 & 0.658 & 27.9 & Inf & $1.45 \mathrm{E}-08$ & $2.67 \mathrm{E}-09$ & $1.83 \mathrm{E}-06$ \\
\hline b.tot.241 & 0.928 & 19.8 & Inf & $-2.06 \mathrm{E}-05$ & $-3.79 \mathrm{E}-06$ & $3.69 \mathrm{E}+00$ \\
\hline Cor & $\mathrm{NA}$ & $\mathrm{NA}$ & $\mathrm{NA}$ & $\mathrm{NA}$ & $-1.42 \mathrm{E}-07$ & $-5.20 \mathrm{E}-03$ \\
\hline Result & 0.00218 & 0.905 & 63.9 & NA & NA & NA \\
\hline${ }^{242} \mathrm{Pu} /{ }^{239} \mathrm{Pu}$ & & & & & & \\
\hline R.242.239 & 0.00103 & 0.88 & 37 & $1.04 \mathrm{E}+00$ & $9.38 \mathrm{E}-06$ & $4.35 \mathrm{E}+01$ \\
\hline CF.242.239 & 1.04 & 0.938 & 31 & $1.03 \mathrm{E}-03$ & $1.00 \mathrm{E}-05$ & $4.94 \mathrm{E}+01$ \\
\hline b.tot.239 & 0.658 & 27.9 & Inf & $6.98 \mathrm{E}-09$ & $1.28 \mathrm{E}-09$ & $8.10 \mathrm{E}-07$ \\
\hline b.tot.242 & 6.71 & 2.74 & Inf & $-2.06 \mathrm{E}-05$ & $-3.80 \mathrm{E}-06$ & $7.12 \mathrm{E}+00$ \\
\hline Cor & $\mathrm{NA}$ & $\mathrm{NA}$ & $\mathrm{NA}$ & NA & $-9.84 \mathrm{E}-08$ & $-4.79 \mathrm{E}-03$ \\
\hline Result & 0.00107 & 1.33 & 77.1 & NA & NA & NA \\
\hline
\end{tabular}




\begin{tabular}{|l|l|l|l|l|l|l|}
\hline \multicolumn{2}{|l|}{ Pu GUM Tables For Sample 85395 (50846) } & & & \\
\hline${ }^{240} \mathrm{Pu} /{ }^{239} \mathrm{Pu}$ & & & & & & \\
\hline Quantity & Est & $\%$ RE & dof & Sensit & Uncert & Index \\
\hline R.240.239 & 0.0734 & 0.173 & 36.9 & $1.00 \mathrm{E}+00$ & $1.27 \mathrm{E}-04$ & $8.99 \mathrm{E}+01$ \\
\hline CF.240.239 & 1 & 0.0576 & 31 & $7.34 \mathrm{E}-02$ & $4.23 \mathrm{E}-05$ & $1.00 \mathrm{E}+01$ \\
\hline b.tot.239 & 0.697 & 28.3 & Inf & $4.02 \mathrm{E}-07$ & $7.94 \mathrm{E}-08$ & $3.52 \mathrm{E}-05$ \\
\hline b.tot.240 & 4.18 & 4.76 & Inf & $-1.62 \mathrm{E}-05$ & $-3.23 \mathrm{E}-06$ & $5.83 \mathrm{E}-02$ \\
\hline Cor & NA & NA & NA & NA & $-7.13 \mathrm{E}-07$ & $-2.84 \mathrm{E}-03$ \\
\hline Result & 0.0735 & 0.182 & 44.9 & NA & NA & NA \\
\hline${ }^{241} \mathrm{Pu} /{ }^{239} \mathrm{Pu}$ & & & & & & \\
\hline R.241.239 & 0.000764 & 0.642 & 36.8 & $1.03 \mathrm{E}+00$ & $5.05 \mathrm{E}-06$ & $3.78 \mathrm{E}+01$ \\
\hline CF.241.239 & 1.03 & 0.715 & 31 & $7.64 \mathrm{E}-04$ & $5.63 \mathrm{E}-06$ & $4.70 \mathrm{E}+01$ \\
\hline b.tot.239 & 0.697 & 28.3 & Inf & $4.13 \mathrm{E}-09$ & $8.15 \mathrm{E}-10$ & $9.84 \mathrm{E}-07$ \\
\hline b.tot.241 & 1.01 & 19.5 & Inf & $-1.62 \mathrm{E}-05$ & $-3.20 \mathrm{E}-06$ & $1.52 \mathrm{E}+01$ \\
\hline Cor & NA & NA & NA & NA & $-7.23 \mathrm{E}-08$ & $-7.73 \mathrm{E}-03$ \\
\hline Result & 0.000787 & 1.04 & 90.8 & NA & NA & NA \\
\hline${ }^{242} \mathrm{Pu} /{ }^{239} \mathrm{Pu}$ & & & & & & \\
\hline R.242.239 & 0.00021 & 1.46 & 35.2 & $1.04 \mathrm{E}+00$ & $3.18 \mathrm{E}-06$ & $4.11 \mathrm{E}+01$ \\
\hline CF.242.239 & 1.04 & 0.938 & 31 & $2.10 \mathrm{E}-04$ & $2.04 \mathrm{E}-06$ & $1.69 \mathrm{E}+01$ \\
\hline b.tot.239 & 0.697 & 28.3 & Inf & $1.11 \mathrm{E}-09$ & $2.19 \mathrm{E}-10$ & $1.95 \mathrm{E}-07$ \\
\hline b.tot.242 & 7.71 & 2.56 & Inf & $-1.62 \mathrm{E}-05$ & $-3.21 \mathrm{E}-06$ & $4.20 \mathrm{E}+01$ \\
\hline Cor & NA & NA & NA & NA & $-3.74 \mathrm{E}-08$ & $-5.70 \mathrm{E}-03$ \\
\hline Result & 0.000218 & 2.28 & 175 & NA & NA & NA \\
\hline
\end{tabular}




\begin{tabular}{|l|l|l|l|l|l|l|}
\hline \multicolumn{2}{|l|}{ Pu GUM Tables For Sample 85394 (3098) } & & & \\
\hline${ }^{240} \mathrm{Pu}{ }^{239} \mathrm{Pu}$ & & & & & & \\
\hline Quantity & Est & $\%$ RE & dof & Sensit & Uncert & Index \\
\hline R.240.239 & 0.137 & 0.172 & 44 & $1.00 \mathrm{E}+00$ & $2.36 \mathrm{E}-04$ & $8.99 \mathrm{E}+01$ \\
\hline CF.240.239 & 1 & 0.0576 & 31 & $1.37 \mathrm{E}-01$ & $7.90 \mathrm{E}-05$ & $1.01 \mathrm{E}+01$ \\
\hline b.tot.239 & 0.603 & 29.3 & Inf & $1.43 \mathrm{E}-06$ & $2.52 \mathrm{E}-07$ & $1.02 \mathrm{E}-04$ \\
\hline b.tot.240 & 2.69 & 6.58 & Inf & $-3.09 \mathrm{E}-05$ & $-5.46 \mathrm{E}-06$ & $4.81 \mathrm{E}-02$ \\
\hline Cor & NA & NA & NA & NA & $-1.66 \mathrm{E}-06$ & $-4.42 \mathrm{E}-03$ \\
\hline Result & 0.137 & 0.182 & 53.5 & NA & NA & NA \\
\hline${ }^{241} \mathrm{Pu} /{ }^{239} \mathrm{Pu}$ & & & & & & \\
\hline R.241.239 & 0.00245 & 0.593 & 44.1 & $1.03 \mathrm{E}+00$ & $1.50 \mathrm{E}-05$ & $3.86 \mathrm{E}+01$ \\
\hline CF.241.239 & 1.03 & 0.715 & 31 & $2.45 \mathrm{E}-03$ & $1.81 \mathrm{E}-05$ & $5.63 \mathrm{E}+01$ \\
\hline b.tot.239 & 0.603 & 29.3 & Inf & $2.53 \mathrm{E}-08$ & $4.46 \mathrm{E}-09$ & $3.41 \mathrm{E}-06$ \\
\hline b.tot.241 & 0.791 & 22.3 & Inf & $-3.09 \mathrm{E}-05$ & $-5.44 \mathrm{E}-06$ & $5.09 \mathrm{E}+00$ \\
\hline Cor & NA & NA & NA & NA & $-2.20 \mathrm{E}-07$ & $-8.34 \mathrm{E}-03$ \\
\hline Result & 0.00253 & 0.953 & 73.5 & NA & NA & NA \\
\hline${ }^{242} \mathrm{Pu} /{ }^{239} \mathrm{Pu}$ & & & & & & \\
\hline R.242.239 & 0.00136 & 0.93 & 43.4 & $1.04 \mathrm{E}+00$ & $1.31 \mathrm{E}-05$ & $4.57 \mathrm{E}+01$ \\
\hline CF.242.239 & 1.04 & 0.938 & 31 & $1.36 \mathrm{E}-03$ & $1.32 \mathrm{E}-05$ & $4.64 \mathrm{E}+01$ \\
\hline b.tot.239 & 0.603 & 29.3 & Inf & $1.39 \mathrm{E}-08$ & $2.45 \mathrm{E}-09$ & $1.60 \mathrm{E}-06$ \\
\hline b.tot.242 & 4.81 & 3.67 & Inf & $-3.09 \mathrm{E}-05$ & $-5.45 \mathrm{E}-06$ & $7.91 \mathrm{E}+00$ \\
\hline Cor & NA & NA & NA & NA & $-1.63 \mathrm{E}-07$ & $-7.10 \mathrm{E}-03$ \\
\hline Result & 0.00141 & 1.38 & 85 & NA & NA & NA \\
\hline
\end{tabular}

\begin{tabular}{|l|l|l|l|l|l|}
\hline \multicolumn{2}{|l|}{ Summary Table For Pu } & & & & \\
\hline${ }^{240} \mathrm{Pu} /{ }^{239} \mathrm{Pu}$ & & & & & \\
\hline Sample & Est & $\% \mathrm{RE}$ & dof & K.factor & $95 \%$ CB \\
\hline 85393 & 0.125 & 0.1662 & 47.49 & 2.011 & 0.0004179 \\
\hline 85395 & 0.07348 & 0.1821 & 44.93 & 2.014 & 0.0002695 \\
\hline 85394 & 0.1373 & 0.1815 & 53.49 & 2.005 & 0.0004996 \\
\hline${ }^{241} \mathrm{Pu} /{ }^{239} \mathrm{Pu}$ & & & & & \\
\hline 85393 & 0.002175 & 0.9053 & 63.88 & 1.998 & $3.93 \mathrm{E}-05$ \\
\hline 85395 & 0.0007875 & 1.043 & 90.8 & 1.986 & $1.63 \mathrm{E}-05$ \\
\hline 85394 & 0.00253 & 0.9533 & 73.51 & 1.993 & $4.81 \mathrm{E}-05$ \\
\hline${ }^{242} \mathrm{Pu} /{ }^{239} \mathrm{Pu}$ & & & & & \\
\hline 85393 & 0.001066 & 1.334 & 77.06 & 1.991 & $2.83 \mathrm{E}-05$ \\
\hline 85395 & 0.0002175 & 2.279 & 175.1 & 1.974 & $9.78 \mathrm{E}-06$ \\
\hline 85394 & 0.001408 & 1.376 & 85.02 & 1.988 & $3.85 \mathrm{E}-05$ \\
\hline
\end{tabular}




\begin{tabular}{|l|l|l|l|l|l|l|}
\hline \multicolumn{2}{|l|}{ U GUM Tables For Sample 85238 (27008) } & & & \\
\hline${ }^{236} \mathrm{U}^{238} \mathrm{U}$ & & & & & & \\
\hline Quantity & Est & $\%$ RE & dof & Sensit & Uncert & Index \\
\hline R.236.238 & 0.000364 & 0.566 & 34.6 & $1.00 \mathrm{E}+00$ & $2.06 \mathrm{E}-06$ & $4.05 \mathrm{E}+01$ \\
\hline CF.236.238 & 1 & 0.5 & Inf & $3.64 \mathrm{E}-04$ & $1.82 \mathrm{E}-06$ & $3.16 \mathrm{E}+01$ \\
\hline b.tot.238 & 0.722 & 48.8 & Inf & $4.42 \mathrm{E}-10$ & $1.56 \mathrm{E}-10$ & $2.31 \mathrm{E}-07$ \\
\hline b.tot.236 & 0.722 & 48.8 & Inf & $-4.86 \mathrm{E}-06$ & $-1.71 \mathrm{E}-06$ & $2.79 \mathrm{E}+01$ \\
\hline Cor & NA & NA & NA & NA & $-2.31 \mathrm{E}-08$ & $-5.08 \mathrm{E}-03$ \\
\hline Result & 0.000364 & 0.89 & 211 & NA & NA & NA \\
\hline${ }^{235}{ }^{236} \mathrm{U}$ & & & & & & \\
\hline R.235.238 & 0.00505 & 0.286 & 34.8 & $9.96 \mathrm{E}-01$ & $1.44 \mathrm{E}-05$ & $9.17 \mathrm{E}+01$ \\
\hline CF.235.238 & 0.996 & 0.079 & 8 & $5.05 \mathrm{E}-03$ & $3.97 \mathrm{E}-06$ & $7.02 \mathrm{E}+00$ \\
\hline b.tot.238 & 0.722 & 48.8 & Inf & $6.13 \mathrm{E}-09$ & $2.16 \mathrm{E}-09$ & $2.07 \mathrm{E}-06$ \\
\hline b.tot.235 & 0.722 & 48.8 & Inf & $-4.86 \mathrm{E}-06$ & $-1.71 \mathrm{E}-06$ & $1.30 \mathrm{E}+00$ \\
\hline Cor & NA & NA & NA & NA & $-8.60 \mathrm{E}-08$ & $-3.29 \mathrm{E}-03$ \\
\hline Result & 0.00503 & 0.298 & 40.4 & NA & NA & NA \\
\hline${ }^{234} \mathrm{U} /{ }^{238} \mathrm{U}$ & & & & & & \\
\hline R.234.238 & 0.000223 & 0.543 & 34.6 & $9.85 \mathrm{E}-01$ & $1.19 \mathrm{E}-06$ & $2.93 \mathrm{E}+01$ \\
\hline CF.234.238 & 0.985 & 0.326 & 8 & $2.23 \mathrm{E}-04$ & $7.17 \mathrm{E}-07$ & $1.05 \mathrm{E}+01$ \\
\hline b.tot.238 & 0.722 & 48.8 & Inf & $2.71 \mathrm{E}-10$ & $9.56 \mathrm{E}-11$ & $1.87 \mathrm{E}-07$ \\
\hline b.tot.234 & 0.722 & 48.8 & Inf & $-4.86 \mathrm{E}-06$ & $-1.71 \mathrm{E}-06$ & $6.02 \mathrm{E}+01$ \\
\hline Cor & NA & NA & NA & NA & $-1.81 \mathrm{E}-08$ & $-6.72 \mathrm{E}-03$ \\
\hline Result & 0.00022 & 1 & 259 & NA & NA & NA \\
\hline
\end{tabular}

\begin{tabular}{|l|l|l|l|l|l|l|}
\hline \multicolumn{2}{|l|}{ U GUM Tables For Sample 85239 (30986) } & & & \\
\hline${ }^{236} \mathrm{U}^{238} \mathrm{U}$ & & & & & & \\
\hline Quantity & Est & $\%$ RE & dof & Sensit & Uncert & Index \\
\hline R.236.238 & 0.0004 & 0.488 & 34.6 & $1.00 \mathrm{E}+00$ & $1.95 \mathrm{E}-06$ & $4.86 \mathrm{E}+01$ \\
\hline CF.236.238 & 1 & 0.5 & Inf & $4.00 \mathrm{E}-04$ & $2.00 \mathrm{E}-06$ & $5.10 \mathrm{E}+01$ \\
\hline b.tot.238 & 0.407 & 9.18 & Inf & $4.96 \mathrm{E}-10$ & $1.86 \mathrm{E}-11$ & $4.38 \mathrm{E}-09$ \\
\hline b.tot.236 & 0.407 & 9.18 & Inf & $-4.96 \mathrm{E}-06$ & $-1.86 \mathrm{E}-07$ & $4.38 \mathrm{E}-01$ \\
\hline Cor & NA & NA & NA & NA & $-2.62 \mathrm{E}-09$ & $-8.76 \mathrm{E}-05$ \\
\hline Result & 0.0004 & 0.7 & 146 & NA & NA & NA \\
\hline${ }^{235} \mathrm{U}{ }^{236} \mathrm{U}$ & & & & & & \\
\hline R.235.238 & 0.0048 & 0.234 & 34.6 & $9.96 \mathrm{E}-01$ & $1.12 \mathrm{E}-05$ & $8.98 \mathrm{E}+01$ \\
\hline CF.235.238 & 0.996 & 0.079 & 8 & $4.80 \mathrm{E}-03$ & $3.78 \mathrm{E}-06$ & $1.02 \mathrm{E}+01$ \\
\hline b.tot.238 & 0.407 & 9.18 & Inf & $5.96 \mathrm{E}-09$ & $2.23 \mathrm{E}-10$ & $3.55 \mathrm{E}-08$ \\
\hline b.tot.235 & 0.407 & 9.18 & Inf & $-4.96 \mathrm{E}-06$ & $-1.86 \mathrm{E}-07$ & $2.46 \mathrm{E}-02$ \\
\hline Cor & NA & NA & NA & NA & $-9.09 \mathrm{E}-09$ & $-5.91 \mathrm{E}-05$ \\
\hline Result & 0.00479 & 0.247 & 40.7 & NA & NA & NA \\
\hline${ }^{234} \mathrm{U}^{238} \mathrm{U}$ & & & & & & \\
\hline R.234.238 & 0.000246 & 0.69 & 34.6 & $9.85 \mathrm{E}-01$ & $1.67 \mathrm{E}-06$ & $8.10 \mathrm{E}+01$ \\
\hline CF.234.238 & 0.985 & 0.326 & 8 & $2.46 \mathrm{E}-04$ & $7.89 \mathrm{E}-07$ & $1.80 \mathrm{E}+01$ \\
\hline b.tot.238 & 0.407 & 9.18 & Inf & $3.05 \mathrm{E}-10$ & $1.14 \mathrm{E}-11$ & $3.76 \mathrm{E}-09$ \\
\hline b.tot.234 & 0.407 & 9.18 & Inf & $-4.96 \mathrm{E}-06$ & $-1.86 \mathrm{E}-07$ & $9.98 \mathrm{E}-01$ \\
\hline Cor & NA & NA & NA & NA & $-2.06 \mathrm{E}-09$ & $-1.23 \mathrm{E}-04$ \\
\hline Result & 0.000242 & 0.767 & 43.5 & NA & NA & NA \\
\hline
\end{tabular}




\begin{tabular}{|l|l|l|l|l|l|l|}
\hline \multicolumn{2}{|l|}{ U GUM Tables For Sample 85240 (50846) } & & & \\
\hline${ }^{236} \mathrm{U}^{238} \mathrm{U}$ & & & & & & \\
\hline Quantity & Est & $\%$ RE & dof & Sensit & Uncert & Index \\
\hline R.236.238 & 0.000231 & 0.728 & 34.1 & $1.00 \mathrm{E}+00$ & $1.68 \mathrm{E}-06$ & $6.20 \mathrm{E}+01$ \\
\hline CF.236.238 & 1 & 0.5 & Inf & $2.31 \mathrm{E}-04$ & $1.16 \mathrm{E}-06$ & $2.92 \mathrm{E}+01$ \\
\hline b.tot.238 & 0.481 & 23.2 & Inf & $3.28 \mathrm{E}-10$ & $3.66 \mathrm{E}-11$ & $2.93 \mathrm{E}-08$ \\
\hline b.tot.236 & 0.481 & 23.2 & Inf & $-5.68 \mathrm{E}-06$ & $-6.33 \mathrm{E}-07$ & $8.77 \mathrm{E}+00$ \\
\hline Cor & NA & NA & NA & NA & $-6.81 \mathrm{E}-09$ & $-1.01 \mathrm{E}-03$ \\
\hline Result & 0.000231 & 0.925 & 88.8 & NA & NA & NA \\
\hline${ }^{235} \mathrm{U}{ }^{236} \mathrm{U}$ & & & & & & \\
\hline R.235.238 & 0.00582 & 0.204 & 34.2 & $9.96 \mathrm{E}-01$ & $1.18 \mathrm{E}-05$ & $8.67 \mathrm{E}+01$ \\
\hline CF.235.238 & 0.996 & 0.079 & 8 & $5.82 \mathrm{E}-03$ & $4.58 \mathrm{E}-06$ & $1.30 \mathrm{E}+01$ \\
\hline b.tot.238 & 0.481 & 23.2 & Inf & $8.27 \mathrm{E}-09$ & $9.23 \mathrm{E}-10$ & $5.27 \mathrm{E}-07$ \\
\hline b.tot.235 & 0.481 & 23.2 & Inf & $-5.68 \mathrm{E}-06$ & $-6.33 \mathrm{E}-07$ & $2.48 \mathrm{E}-01$ \\
\hline Cor & NA & NA & NA & NA & $-3.42 \mathrm{E}-08$ & $-7.24 \mathrm{E}-04$ \\
\hline Result & 0.0058 & 0.219 & 41.5 & NA & NA & NA \\
\hline${ }^{234} \mathrm{U} /{ }^{238} \mathrm{U}$ & & & & & & \\
\hline R.234.238 & 0.000109 & 0.968 & 33.9 & $9.85 \mathrm{E}-01$ & $1.04 \mathrm{E}-06$ & $6.74 \mathrm{E}+01$ \\
\hline CF.234.238 & 0.985 & 0.326 & 8 & $1.09 \mathrm{E}-04$ & $3.51 \mathrm{E}-07$ & $7.65 \mathrm{E}+00$ \\
\hline b.tot.238 & 0.481 & 23.2 & Inf & $1.55 \mathrm{E}-10$ & $1.73 \mathrm{E}-11$ & $1.86 \mathrm{E}-08$ \\
\hline b.tot.234 & 0.481 & 23.2 & Inf & $-5.68 \mathrm{E}-06$ & $-6.33 \mathrm{E}-07$ & $2.49 \mathrm{E}+01$ \\
\hline Cor & NA & NA & NA & NA & $-4.68 \mathrm{E}-09$ & $-1.36 \mathrm{E}-03$ \\
\hline Result & 0.000108 & 1.18 & 70.6 & NA & NA & NA \\
\hline
\end{tabular}

\begin{tabular}{|l|l|l|l|l|l|}
\hline \multicolumn{2}{|l|}{ Summary Table For U } & & & & \\
\hline${ }^{236} \mathrm{U} /{ }^{238} \mathrm{U}$ & & & & & \\
\hline Sample & Est & $\%$ RE & dof & K.factor & $95 \%$ CB \\
\hline 85238 & 0.0003641 & 0.8899 & 210.8 & 1.971 & $6.39 \mathrm{E}-06$ \\
\hline 85239 & 0.0004004 & 0.7004 & 146.4 & 1.976 & $5.54 \mathrm{E}-06$ \\
\hline 85240 & 0.0002312 & 0.9249 & 88.76 & 1.987 & $4.25 \mathrm{E}-06$ \\
\hline${ }^{235} \mathrm{U} /{ }^{236} \mathrm{U}$ & & & & & \\
\hline 85238 & 0.005029 & 0.2982 & 40.36 & 2.021 & $3.03 \mathrm{E}-05$ \\
\hline 85239 & 0.004786 & 0.2472 & 40.7 & 2.02 & $2.39 \mathrm{E}-05$ \\
\hline 85240 & 0.005802 & 0.219 & 41.46 & 2.019 & $2.57 \mathrm{E}-05$ \\
\hline${ }^{234} \mathrm{U} /{ }^{238} \mathrm{U}$ & & & & & \\
\hline 85238 & 0.00022 & 1.003 & 258.9 & 1.969 & $4.35 \mathrm{E}-06$ \\
\hline 85239 & 0.0002421 & 0.767 & 43.5 & 2.016 & $3.74 \mathrm{E}-06$ \\
\hline 85240 & 0.0001077 & 1.178 & 70.6 & 1.994 & $2.53 \mathrm{E}-06$ \\
\hline
\end{tabular}




\subsection{Summary}

Most $\mathrm{U}$ and $\mathrm{Pu}$ ratio estimates determined in the GUM analyses compare very well, within analytical uncertainty, with ratios determined by the in-house data reduction program. Comparisons are summarized in the table below. Data values with 2 sigma are corrected for mass bias and are from the inhouse data reduction routine, and values with 'RE' (relative error) are estimates of $95 \%$ confidence intervals from GUM uncertainty analysis which are also corrected for measurement mass bias. The inhouse routine may underestimate uncertainty and error, and the 'RE' determined by the GUM analysis is typically slightly higher than the 2-sigma values reported for some ratios.

\begin{tabular}{|c|c|c|c|c|c|c|c|}
\hline sample & PNNL No. & ${ }^{234} \mathrm{U} /{ }^{238} \mathrm{U}$ & 2 sigma & ${ }^{235} \mathrm{U} /{ }^{236} \mathrm{U}$ & 2 sigma & ${ }^{236} \mathrm{U} /{ }^{238} \mathrm{U}$ & 2 sigma \\
\hline 27008 & 85238 & 0.000221 & 0.000002 & 0.005042 & 0.000026 & 0.000365 & 0.000004 \\
\hline 30986 & 85239 & 0.000242 & 0.000003 & 0.004784 & 0.000017 & 0.000400 & 0.000004 \\
\hline 50846 & 85240 & 0.000107 & 0.000003 & 0.005776 & 0.000020 & 0.000231 & 0.000003 \\
\hline & & & $\mathrm{RE}$ & & $\mathrm{RE}$ & & $\mathrm{RE}$ \\
\hline 27008 & 85238 & 0.00022 & 0.000004 & 0.00503 & 0.000030 & 0.000364 & 0.000006 \\
\hline 30986 & 85239 & 0.00024 & 0.000004 & 0.00479 & 0.000024 & 0.000400 & 0.000006 \\
\hline 50846 & 85240 & 0.000108 & 0.000003 & 0.0058 & 0.000026 & 0.000231 & 0.000004 \\
\hline & & & & & & & \\
\hline 1 & & ${ }^{240} \mathrm{Pu} /{ }^{239} \mathrm{Pu}$ & 2 sigma & ${ }^{241} \mathrm{Pu} /{ }^{239} \mathrm{Pu}$ & 2 sigma & ${ }^{242} \mathrm{Pu} /{ }^{239} \mathrm{Pu}$ & $2 \mathrm{sigma}$ \\
\hline 27008 & 85393 & 0.12505 & 0.00030 & 0.002190 & 0.000020 & 0.001081 & 0.000016 \\
\hline 30986 & 85395 & 0.13741 & 0.00031 & 0.002545 & 0.000021 & 0.001419 & 0.000017 \\
\hline 50846 & 85394 & 0.07334 & 0.00018 & 0.000787 & 0.000012 & 0.000218 & 0.000006 \\
\hline 2 & & & $\mathrm{RE}$ & & $\mathrm{RE}$ & & $\mathrm{RE}$ \\
\hline 27008 & 85393 & 0.12500 & 0.00042 & 0.002180 & 0.000042 & 0.001030 & 0.000028 \\
\hline 30986 & 85395 & 0.13700 & 0.00050 & 0.002530 & 0.000048 & 0.001410 & 0.000038 \\
\hline 50846 & 85394 & 0.07350 & 0.00026 & 0.000787 & 0.000016 & 0.000218 & 0.000010 \\
\hline
\end{tabular}





\section{Distribution List}

No. of

Copies

\section{OFFSITE}

2 William C. O'Connor

U.S. Dept. of Energy

NA-241/L'Enfant Plaza Building

1000 Independence Avenue, S.W.

Washington, DC 20585-1615

1 David S. Simon

NIST

Bldg. 222, Room A113

Gaithersburg, MD 20899-0001

1 Steve Goldberg

U.S. Dept. of Energy

New Brunswick Laboratory

9800 S. Cass Avenue, Bldg. 350

Argonne, IL 60439-4899

\section{ONSITE}

5 Pacific Northwest National Laboratory

DC Gerlach (1) P7-07

PG Heasler (1) K6-08

BD Reid (1) K8-34

Information Release (2) P8-55

Distr. 1 